\title{
Properties improvement of titanium alloys scaffolds in bone tissue engineering: a literature review
}

\author{
Weiyang Zuo", Lingjia Yu", Jisheng Lin, Yong Yang, Qi Fei \\ Department of Orthopedics, Beijing Friendship Hospital, Capital Medical University, Beijing, China \\ Contributions: (I) Conception and design: W Zuo, L Yu; (II) Administrative support: Q Fei, Y Yang; (III) Provision of study materials or patients: \\ None; (IV) Collection and assembly of data: W Zuo, J Lin; (V) Data analysis and interpretation: W Zuo, L Yu; (VI) Manuscript writing: All authors; \\ (VII) Final approval of manuscript: All authors. \\ "These authors contributed equally to this work. \\ Correspondence to: Qi Fei. Department of Orthopaedics, Beijing Friendship Hospital, Capital Medical University, Beijing 100050, China. \\ Email: spinefei@ccmu.edu.cn.
}

\begin{abstract}
Owing to their excellent biocompatibility and corrosion-resistant properties, titanium (Ti) (and its alloy) are essential artificial substitute biomaterials for orthopedics. However, flaws, such as weak osteogenic induction ability and higher Young's modulus, have been observed during clinical application. As a result, short- and long-term postoperative follow-up has found that several complications have occurred. For decades, scientists have exerted efforts to compensate for these deficiencies. Different modification methods have been investigated, including changing alloy contents, surface structure transformation, threedimensional (3D) structure transformation, coating, and surface functionalization technologies. The cellsurface interaction effect and imitation of the natural 3D bone structure are the two main mechanisms of these improved methods. In recent years, significant progress has been made in materials science research methods, including thorough research of titanium alloys of different compositions, precise surface pattern control technology, controllable 3D structure construction technology, improvement of coating technologies, and novel concepts of surface functionalization. These improvements facilitate the possibility for further research in the field of bone tissue engineering. Although the underlying mechanism is still not fully understood, these studies still have some implications for clinical practice. Therefore, for the direction of further research, it is beneficial to summarize these studies according to the basal method used. This literature review aimed to classify these technologies, thereby providing beginners with a preliminary understanding of the field.
\end{abstract}

Keywords: Titanium (Ti); 3D structure; biomaterials; orthopedics

Submitted Dec 27, 2020. Accepted for publication May 28, 2021.

doi: $10.21037 /$ atm-20-8175

View this article at: https://dx.doi.org/10.21037/atm-20-8175

\section{Introduction}

Since the mid-nineteenth century, the repair of mass bone defects has become a focal point of research in orthopedics and oral and maxillofacial surgery. The most commonly used materials are metal, biological ceramics, and highmolecular polymers. With modern medicine and materials development, some novel biological ceramics and bio-glass are superior to traditional metals in some aspects. However, given their tenacity, durability, and cost-effectiveness, metallic prostheses are still the first choice for orthopedic surgery.

As the most commonly used material, titanium (Ti) is a silver-white metal that exhibits the following characteristics: high strength, lightweight, strong plasticity, corrosion resistance, and good biocompatibility (1). Since the invention of the magnesium reduction process to produce pure titanium, it has been widely used in medicine, 


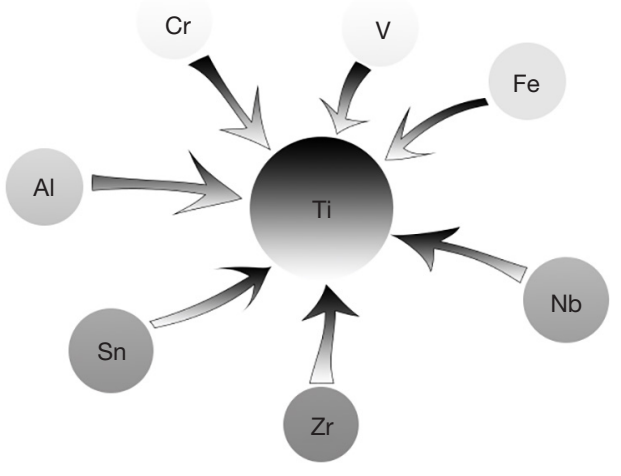

Figure 1 Component change of the titanium alloy. The materials science researchers dedicate to add elements in different proportions into titanium, which could construct different alloy phases and get different physical or bioactive properties.

aerospace, manufacturing, and other industries. In the healthcare industry, titanium is non-toxic, biocompatible, and corrosion resistant, making it a common material for manufacture artificial bone defect fillers and internal fixation screw systems (2). However, further development of research and application has gradually revealed some disadvantages. Firstly, the osteogenic induction ability of conventional titanium alloys is weak, which may lead to the formation of fibrous connective tissue film on the bonemetal contact surface, thus affecting bone regeneration and integration (3). Secondly, Young's modulus of titanium is higher than that of natural bone, which directly causes a degree of bone resorption around the prosthesis during daily activities postoperatively, thereby increasing the risk of osteolysis, resorption, postoperative complications, and secondary surgery $(4,5)$. Thirdly, recent research has shown that the wear debris of titanium could induce local biological reactions, including inflammation, apoptosis, and necrosis, resulting in associated complications $(6,7)$. Moreover, although its excellent durability is an essential feature as a bone-tissue-engineering material, this characteristic means that a second operation may be required for removal to avoid complications like osteolysis and non-union.

Over the last decade, several methodologies have been explored to reform the properties of titanium alloys. In this study, we reviewed relevant studies from the PubMed database from the last 5 years and attempted to predict the development prospects for the property improvement technology of titanium alloys.

Data sources: PubMed database searches were completed using the following MeSH terms: Titanium, Bone Formation, Ossification, Ossifications, Osteoclastogenesis, Osteoclastogeneses. The search included randomized controlled trials, meta-analyses, clinical trials, systematic reviews, clinical practice guidelines, evidence-based medicine, and review articles. Search dates: January 2015 through February 2020.

We present the following article in accordance with the Narrative Review reporting checklist (available at https:// dx.doi.org/10.21037/atm-20-8175).

\section{Alloy composition change}

In 1940, Bothe et al. (8) first used titanium in medical experiments and confirmed its excellent biocompatibility. This study directly led to the primary use of pure titanium as the research object of bone tissue engineering over the next 30 years, with advantages such as good biocompatibility and strong corrosion-resistance being discovered (9). In 1952, Brånemark et al. (10) used pure titanium to construct a metal cage spiral that was inserted into a rabbit bone. A few months later, they found that it was closely bound to the bone and proposed the concept of "osseointegration", which made titanium a popular material for surgical operations.

In the following decade, researchers identified that the high Young's modulus of pure titanium could result in the shade with bone stress, leading to the dissolution of bone around the implant, aseptic necrosis as fiber, and coated formation which causes loosening of the prosthesis. Moreover, metal fatigue occurred in the body's fluid environment due to the lower static stress strength of pure titanium. Adding other metal or non-metal elements to compensate for these shortcomings became a popular material science research direction (Figure 1). In 1982, Ti6Al4V was used to produce an artificial joint prosthesis and provided a new idea for using a novel titanium alloy. Initially, niobium $(\mathrm{Nb})$ became a substitute metal element for vanadium $(\mathrm{V})$ and aluminum $(\mathrm{Al})$ in numerous studies due to its lower inherent cytotoxicity (11-15).

Next, although the $\alpha+\beta$ type titanium alloy has reduced Young's modulus to some extent, Young's modulus of human bones is still lower than that of the $\alpha+\beta$ type titanium alloy. Therefore, the $\beta$-type titanium alloy (about 40-60 Giga Pascal, GPA) has attracted extensive attention as a new research object (16-18). Also, the impact of the thermal effect on the alloy phase has been studied for decades. Malinov et al. (19) found that the alloy phase changed from 
$\alpha$ to $\beta$ in a temperature- and proper chemical-controlled environment. Kopova et al. added a small amount of Ferrum (Fe) (0-2 wt.\%) and Silicon (Si) (0-1 wt.\%) to Ti$35 \mathrm{Nb}-7 \mathrm{Zr}-6 \mathrm{Ta}$ in order to regulate Young's modulus and biological properties, and thus, developed a novel $\beta$ type titanium alloy (Ti-35Nb-7Zr-6Ta-2Fe-0.5Si) $(17,20)$.

Due to the different element compositions of the three types of titanium alloy, their manufacturing and casting processes are also markedly different. It is essential to examine the treatment mechanism and method of the $\beta$ type titanium alloy. For example, Plasma Electrolytic Oxidation $(\mathrm{PEO})$ is a traditional surface microstructure treatment technology. In a recent study, Tanase et al. (21) selected various kinds of titanium alloy. They divided them into different experimental groups according to their alloy phase, including pure titanium (CP, $\alpha$ type), titanium (Ti6Al4V, $\alpha+\beta$ type), titanium (Ti13 Nb13Zr, close $\beta$ type), and titanium alloy (Ti45Nb, completely $\beta$ type) groups. They managed the surface microstructure treatment process for each group by PEO and found that this technology could effectively construct a better rough surface on the $\beta$ type alloy compared to the $\alpha$ type alloy. However, the complexity of the manufacturing process and high production price limits its mass production and clinical application. More cost-efficient and convenient production methods need to be explored.

Besides changing the physical properties of the alloy, some metal elements have also been added to the alloy composition to reduce the occurrence of postoperative short- or long-term complications. Xu et al. (22) incorporated copper into the titanium alloy (Ti6Al4V) using Cross Shading and Selective Laser Melting (SLM) technology, which reduced the inflammatory response around the prosthesis and the formation of fibrous tissue capsules. This method played a specific role in promoting bone and angiogenic differentiation. To reduce the probability of bacterial infection around the prosthesis after surgery, Liu et al. (23) added silver to titanium. They obtained an optimal ratio between antibacterial properties and biocompatibility by altering the silver content, which resulted in excellent antibacterial properties for the titanium without damaging human cells.

Over the last decade, many novel medical material engineering technologies that could be combined with other non-metal substances have been developed, which are expected to solve complex problems associated with the metal content of titanium alloys. Spark Plasma Sintering (SPS) was further explored by Fernandez-Garcia et al. (24).
Bioactive ceramics could be combined with titanium to construct novel scaffolds that promote the osteogenic differentiation of stem cells and accelerate the process of bone integration through SPS technology. Kokubun et al. (25) conducted animal experiments with titania-based biological glass alloy (Ti40Zr10Cu34Pd14Sn2) and found that it could effectively minimize the release of metal ions, thus avoiding related cell damage while reducing Young's modulus.

\section{Surface and three-dimensional (3D) structure transformation}

Surface and 3D structure transformation are considered two effective methods to increase the osteogenic induction ability of titanium metal alloy scaffolds, which do not involve changing metal components (Figure 2). Given the mechanism of osteogenic differentiation, compared with the smooth surface, the rough surface can increase the contact surface area between membranes and pseudopodia. The specific morphology could provide various fitting angles, create various peaks or valleys to increase surface hydrophilicity, and reconstruct the structure of proteins to promote absorption, thereby optimizing cell adhesion and proliferation ability. A study on human osteoblasts has shown that rough surfaces have more anchor points and increase the adhesion ability of cells, which could provide better conditions for cell proliferation and increase cell activity (26). Moreover, due to the numerous anchors on the rough surface, cell polarity changes could control the expression of structural proteins, including extracellular matrix protein, cytoskeleton protein, integrin protein, and calcic adhesion protein, etc. Cell polarity changes could also promote the differentiation of bone marrow stem cells into osteoblasts to a certain extent (3). The commonly used methods include groove structure, laser sintering, sandblasting, chemical etching, and anodic oxidation. Based on the acid-etching method, Zheng et al. (27) compared the differences between direct laser sintering and surface sandblasting and showed that direct laser sintering technology was superior to surface sandblasting treatment in terms of epigenetic regulation of osteogenic differentiation.

In the early stages of research, surface treatment methods often fail to control the shape of the generated surface patterns and only use simple physical/chemical methods to generate rough surfaces. With the improvement of technology, it is possible to control the geometric parameters 
A

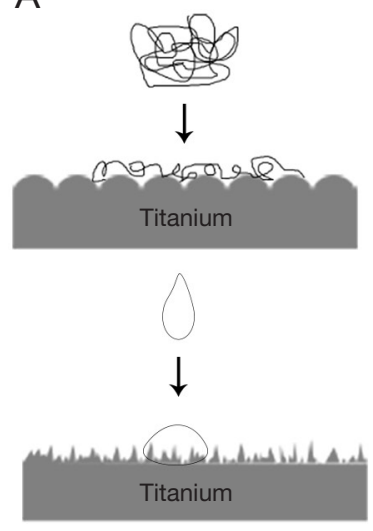

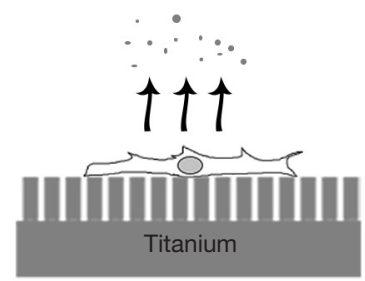

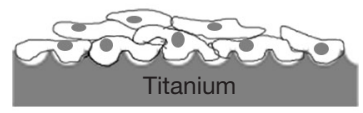

B
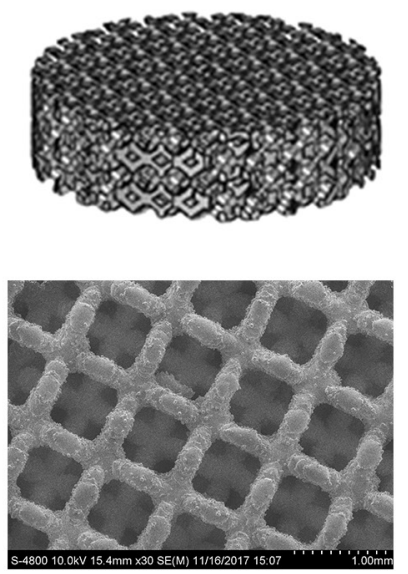

Figure 2 Nano-scale surface modification (A) and 3D structure formation (B). The main structures of nanoscale surface modification are classified as nano-rod, nano-particle, nano-hemisphere and nano-amorphous structure. It could improve titanium alloy properties by promoting protein absorption, cell differentiation, cell proliferation as well as increasing wettability. 3D structure can simulate natural bone structure thus promoting osseointegration, osteogenesis, angiogenesis and reducing Young's modulus of titanium alloy.

and surface microstructure accurately. Zhu et al. (28) reported a combination method of photolithography. They inductively coupled plasma-based dry etching for surface design, which can precisely control the microstructure pattern of the titanium surface and screen appropriate patterns and parameters to achieve the best osteogenesis effect.

At present, further structural treatment primarily includes $3 \mathrm{D}$ printing technology (i.e., selective laser melting and electron beam melting) and nano-level surface structure formation (nanotube array, nano-rod array, etc.). These two technologies both have unique advantages. 3D printing technology provides accurate shape control. Researchers could better simulate the porous structure of human cancellous bone at a 3D level to promote bone growth, thereby helping bone-implant integration. Meanwhile, nano-level surface structure formation can better regulate cell adhesion, proliferation, and differentiation from the nano-level material-cell interaction.

In 2004, Fujibayashi et al. (29) discovered that 3D structures provide a similar growth environment as the human body's natural bone structure compared with twodimensional (2D) structures. Since then, the 3D porous structure has become a particular focus of research. Based on $2 \mathrm{D}$ micro-rough surface, the $3 \mathrm{D}$ porous structure can effectively promote osteogenesis, bone ingrowth, accelerate bone integration, and reduce Young's modulus, thus reducing aseptic bone dissolution (30-33). Meanwhile, the $3 \mathrm{D}$ porous structure promotes the growth of new bone into the scaffold, which promotes bone integration, osteogenesis, and increases angiogenesis, and reduces the occurrence of stent-related complications due to changes in stress and strain (34).

Similarly, Attar et al. produced Ti and Ti-TiB alloy porous structures with different porosity levels. The properties test then demonstrated that $37 \%$ of the porous group showed elastic moduli close to that of human bone (35). However, another study confirmed that porous scaffolds with different shapes or structures have a minimal effect on cellular osteogenesis than the enhanced osteogenic capacity provided by the porous nature itself (36). Hedayati et al. studied the isolated and modulated effects of topological design and material type on mechanical properties. Their results showed that topological modification could significantly influence the porous properties to a greater extent than the material type (37). Also, Zhou et al. (38) verified the osteogenic effect of 3D scaffold-based scaffold on adipose stem cells and confirmed that its mechanism was related to the IGF-1R/protein kinase B (AKT)/Mammalian Target of Rapamycin Complex 1 (mTORC1) cell signaling pathway.

$3 \mathrm{D}$ printing is a new rapid prototyping technology, which has been widely used in the field of tissue engineering research. 3D printing technology has good controllability, making it possible to precisely regulate the porosity and pore diameter of the titanium scaffolds for further research. Some studies have reported that scaffolds with different 
pore sizes, porosity, pore shapes, and pore structures were constructed using this technique to achieve the optimal promotion of physical properties, cell adhesion, proliferation, and osteogenic differentiation (39-43). Kapat et al. (44) used the powder metallurgy method to build Ti6Al4V with different pore sizes and porosities and then measured the pore distribution, mechanical properties, surface roughness, contact angle, and protein adsorption ability. Subsequent in vivo and in vitro experiments determined the optimal impact on cell distribution and differentiation. 3D printing technology typically refers to Electron Beam Melting (EBM) and Selective Laser Melting (SLM) in the metallurgy field. There are differences between these two methods. For example, SLM has a higher resolution ratio and costs more than EBM. Although the operational approaches of these technologies differ, some scientists have found no significant difference in the construction of titanium alloy scaffolds (45).

Micro/nano-meter microstructure morphology construction is another new research direction, which include $3 \mathrm{D}$ printing technology, novel acid corrosion and anodic oxide technology and nano-level surface morphology formation (nano-meter surface pattern, nanotube, and nano-rod arrays). The hydrophilicity, protein adsorption ability, the surface roughness of scaffold are increased, thus promoting cell viability, proliferation, adhesion, and differentiation. Some studies have reported that various forms of nano-meter surface structure improve the biocompatibility and bioactivity of the titanium alloy scaffolds through nanoscale scaffolds-cells interface interaction (46,47). Results reported by Liu et al. (48) may explain the underlying mechanism. Their in vitro experiment indicated that the rough titanium surface could accelerate the osteogenic procession by activating the extracellular regulated protein kinases (ERK1/2)- micro ribonucleic acid (miR)-1827-Osterix signaling pathway. Recent research by Clainche et al. (49) found that the morphology construction could induce osteogenic differentiation and inhibit bacterial infection. They also indicated that the nanostructured surface with sharp nanosheet protrusions could cut the cell membrane of bacteria and reduce bacterial attachment.

Recently, researchers shifted emphasis to the nanometer-micron co-existence mode and found that it was significantly superior for promoting cell osteogenesis than any one of the two structures. Dumas et al. (50) used femtosecond laser technology to construct a nanometermicron notch ripple structure, which significantly increased the cell adhesion and proliferation ability, accelerated cell spread, and increased the cell osteogenesis ability compared to the polished scaffold surface. Gulati et al. (51) combined $3 \mathrm{D}$ printing technology and anodic oxidation technology to construct a micro-surface structure of co-existence micronscale spherical particles and a nano-level vertical tube array, which significantly increased the osteogenic differentiation ability of stem cells. Similarly, Fu et al. (20) used 3D printing technology to construct a regular micron-level repeated ripple and irregular nanoscale branch structure on pure titanium and confirmed that this structure could promote osteogenic differentiation via the wingless/integrated (Wnt)/ $\beta$-catenin signal transduction pathway. However, Zhang et al. (52) proposed an entirely different opinion. In their experiment, although the titanium 3D structure combined with acid-etched could improve in vivo osseointegration ability, the overall osteogenic performance of this surface was not as good as the conventional sandblasted, large-grit, acid-etched surface. These findings suggest that scientists need to explore the mechanisms of cell-surface interaction further.

\section{Coating technology}

Coating technology is one of the most popular methods for modifying the properties of titanium alloy scaffold. Owing to its excellent physical properties, researchers used the physical/chemical/biological method to create various bioactive substances for attaching solids on the titanium alloy scaffold. It is expected that this could solve problems such as poor osteogenic differentiation induction ability, bacterial contamination, osteoporosis, or scaffold-related bone destruction and resorption. At present, the commonly used ingredients mainly include hydroxyapatite (HA) coating, metal and metal oxide coating, or drug/bioactive factor-related coating (Figure 3).

$\mathrm{HA}$ is an essential main inorganic component to natural human bone, which is commonly used as an artificial bone substitute. HA has good biocompatibility, no cytotoxicity, and can be chemically combined with bone tissue. HA can also release calcium, phosphorus, and other osteogenicrelated substances in the human body and be assimilated and absorbed by human bone tissue. In clinical application, surgeons directly inject HA into the bone defect. It has been reported that as the surface coating of titanium alloy, HA can promote cell adhesion, proliferation and increase osteogenic differentiation (53).

Techniques for HA coating were initially developed in the 1990s, including plasma spraying, physical 


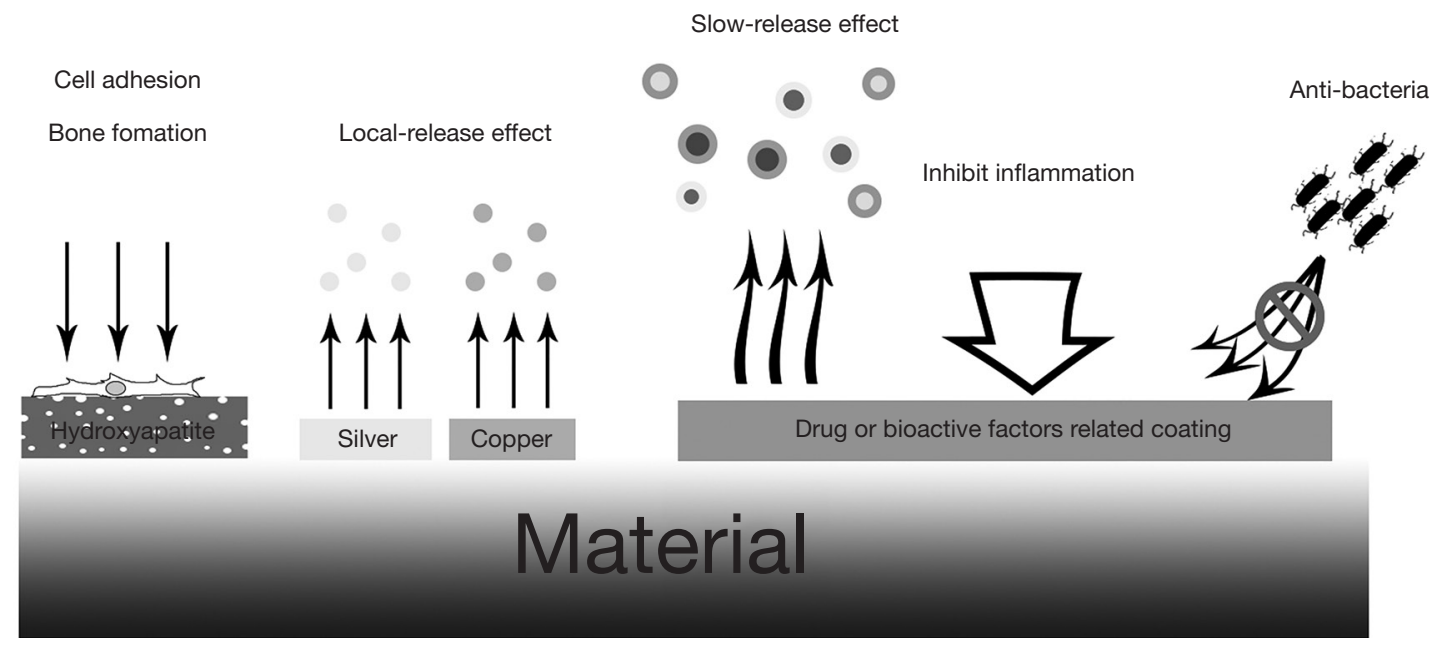

Figure 3 Coating methods of titanium. By selecting the appropriate coating material, the researchers can almost solve any single problem. The hydroxyapatite improves cell adhesion and promotes bone formation. The coating technology can reduce systemic side effects by creating local-release and slow-release effect. The antibiotics or cytokines coating can inhibit bacterial growth and reduce inflammation.

vapor deposition, aerosol-gel technology, and chemical deposition. In recent years, with the development of surface microstructure treatment technology, HA coating can be used with $3 \mathrm{D}$ printing and other technologies to construct nano-level microstructures. However, although it has been shown that the HA coating constructed by this microstructure treatment technology has a more vital osteogenic promotion ability, the coating-scaffold interface adhesion force is poorer than the amorphous coating.

In addition, Jang et al. (54) treated titanium membranes with calcium-phosphate coating and punched holes in the coating film. Their results indicate that this coating could induce bone formation and facilitate a tight combination between the new bone and the titanium surface. The coating of punch holes could reduce bone formation. Some compounds containing carbon and phosphorus, such as organic glass, tricalcium phosphate, calcium phosphate, alendronate sodium, carbon graphene, and calcium-silicon compounds, have been shown to exhibit a more substantial osteogenic differentiation promotion effect than the traditional HA coating and have become a particular focus of research (55-61).

Another kind of candidate for surface coating is metal or metal oxide. Different metals have different properties; for example, tantalum, gray metal with similar physical properties to titanium, can accelerate the natural mineral deposits in body fluids through the activation of the Wnt/ beta and transforming growth factor- $\beta$ (TGF- $\beta$ )/drosophila mothers against decapentaplegic protein (Smad) signaling pathways, which could mediate osteogenesis (62). Wang et al. (63) manufactured a nanotube array coating with tantalum on the titanium alloy scaffold and then seeded adipose-derived stem cells on the coating. The subsequent animal experiment on a rabbit spinal defect model confirmed that it significantly promoted osteogenesis.

Copper and silver have been shown to exhibit significant anti-microbial activity. However, previous studies demonstrated that these ions also resulted in organ toxicity, inhibition of osteogenesis, and bone integration at high concentrations. To avoid these toxic effects, some researchers used a nanoscale metal ion to produce a kind of slow-release coating that inhibits the growth of bacteria but will not damage the properties of the titanium alloy scaffold $(64,65)$.

In a recent study, Zhao et al. (66) reported a novel Tremella-like zinc oxide ( $\mathrm{ZnO})$ /type I collagen (Col-I) composite coating on the titanium surface. They improved the properties of $\mathrm{ZnO}$, which allow a safe, visible yellow light to activate it, and made it adhere to the titanium metal surface, and subsequent experiments confirmed its excellent antibacterial properties and osseointegration ability.

Titanium dioxide is one of the most common and convenient metal oxides. It can be easily obtained chemically or anodic oxidation processes on the pure titanium scaffold. Titanium dioxide coating could change the physical properties and form a micro or nanoscale rough surface structure to some extent, thereby improving the 
hydrophilia and osteogenic differentiation induction ability of scaffold. Its convenience allows it to be combined with other types of titanium property improvement techniques to promote osteogenesis $(67,68)$ further.

Drug/bioactive factor-related coatings are currently the most popular coating technology. This refers to the creation of a slow-release film that carries different drugs depending on the requirement. With further development, this technology could carry traditional drugs and metal ions or seed stem cells. Using the local slow-releasing method, the traditional oral or intravenous administration side effects of medications can be avoided. However, due to the toxicity of local concentration, achieving a stable and controllable slow-release form and selecting an appropriate type of medium that does not cause damage to the property of the scaffold are vital concerns.

The material choice of the coating mediator medium is crucial. Haversath et al. (69) used poly-dl-lactide (PDLLA) carrying rhBMP-2 to form a slow-release coating surface on a titanium alloy, which did not promote bone formation as expected. Researchers hypothesized that PDLLA has a certain degree of inhibition of bone formation, which counteracts the positive effect of rhBMP-2. At present, the commonly used coating media commonly include chitosan, penicillin, calcium-phosphorus compounds, etc., which exhibit good cellular compatibility, no osteogenic inhibition, and could form a slow-releasing structure (70).

Furthermore, the choice of drugs depends on the medical requirement, and the drug dose-effect of controlled-release stents carry side effects and synergy. For example, bone morphogenetic protein-2 (BMP-2) plays a vital role in the process of osteogenesis (71). Recent research has shown that combined with a slow-release coating constructed by chitosan and other medium composition; it can effectively promote the osteogenesis ability of the titanium alloy scaffold (72-74). On the other hand, another study showed that BMP-2 inhibits cell proliferation and adhesion at high concentrations (75).

Metal ions, such as copper, silver, magnesium, strontium, etc., can promote osteogenesis and avoid certain toxicities by being carried with a slow-released coating film (76-79). Van Hengel et al. (80) indicated that the synergistic antibacterial effect could prevent infections by antibioticresistant bacteria. They combined slow-release silver/ zinc nano-particles with a plasma electrolytic oxidation biofunctionalized titanium surface. The results showed that this combination significantly inhibited the growth of methicillin-resistant Staphylococcus aureus and proved to be a fruitful strategy for preventing infection.

Carrying an antibiotic, such as gentamicin, can enhance the accuracy and curative effect of bacterial inhibition to reduce the complications caused by bacterial infection. Furthermore, the bacteria-mediated inflammatory responses could be restrained to avoid the osteogenesis inhibition effect generated by improper inflammatory activation $(81,82)$.

Substances such as alendronate sodium and calcitriol could promote the formation of bone defects around the scaffold and have been shown to play a role in increasing screw stability in animal model experiments of osteoporosis (83-86).

Exosomes are small membranous vesicles containing complex ribose nucleic acid (RNA) and proteins. As a paracrine pathway, they could regulate the function and phenotype of surrounding cells. Zhai et al. (87) extracted and purified exosomes secreted by pre-osteogenic differentiational stem cells to avoid the side effect of stem cell therapeutics. These exosomes were then loaded onto titanium scaffolds. Cell and animal experiments showed that this cell-free scaffold could efficiently regenerate bone tissue, which presents a novel bone regeneration method. Lan et al. (88) reported a similar result further explained the mechanism that this osteogenic induction was achieved partly by activating the RhoA/ROCK signaling pathway.

The remaining studies of carried medicines involved polydopamine $(89,90)$, stem cells, microRNA nanoparticles (91), plasmid deoxyribonucleic acid (DNA) (92), and other drugs that meet the different specific design requirements $(93,94)$.

\section{Functionalization}

Functionalization refers to the use of physical/chemical/ biological surface treatment methods to obtain activating surface properties of titanium alloy, thus improving the bone induction ability and biocompatibility of the alloy without using additional coating materials (Figure 4). The earliest surface functionalization treatment was calcium ion implantation technology reported by Hanawa et al. (95) in 1993. The major mechanism of this technology involves artificial simulation of a natural body environment, thereby inducing spontaneous apatite formation. Researchers introduced calcium ions into pure titanium to form a surface-modified layer containing a variety of calcium and titanium oxides to promote osteogenesis.

Recently, different chemical treatment methods were explored. Alkali heat treatment activation modification refers to submerging the titanium scaffold into a strong 


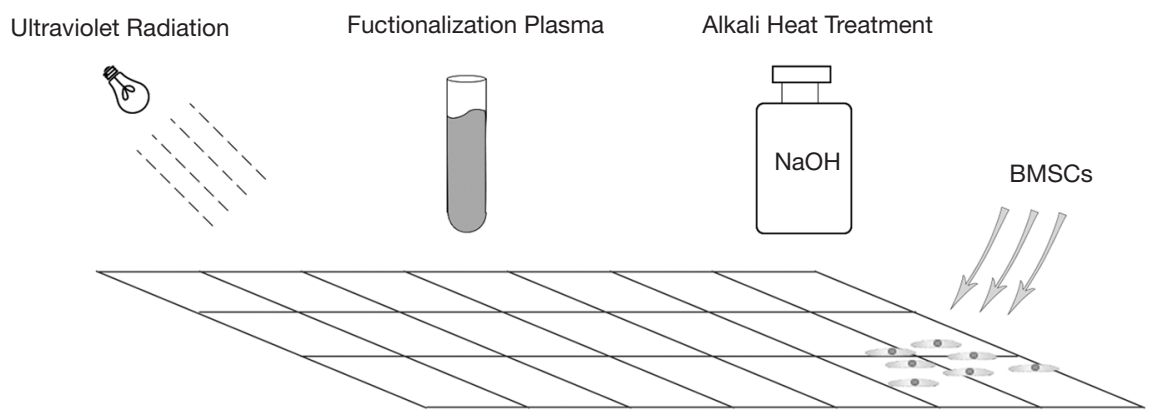

Figure 4 Functionalization. To improve the properties of titanium alloys, the researchers construct activated surfaces by physical/chemical/ biological methods. It could induce apatite formation spontaneously and promote bone formation. The co-culture of exogenous stem cell could stimulate osteogenic differentiation of adjacent somatic cells by secret exosomes.

alkaline liquid for a period of time, and then treating it with high temperature to obtain an active surface, which can release alkaline components into body fluids, thus inducing natural HA deposition and an osteogenic effect (96). Cazzola et al. (97) used tea polyphenols, which are a kind of green tea extract, to functionalize the surface of titanium alloy to distribute hydroxyl groups on the surface, thus increasing the hydrophilicity of the titanium alloy, facilitating the deposition of HA into body fluids and promoting osteogenesis. Kwon et al. innovatively created amine functionalities on the surface of titanium by using a non-thermal atmospheric pressure plasma jet. This method could significantly increase cell adhesion ability and hydrophilicity.

In addition, ultraviolet light-mediated photofunctionalization, which refers to treating the titanium surface with ultraviolet light radiation in a dark room for several days, is a novel research direction of surface functionalization processing. Scientists have found that photofunctionalization can increase the hydrophilic of scaffold and make it absorb the liquid easily (98). Pham et al. (99) treated titanium coins with hydrofluoric acid, thereby creating a fluoride modification $\mathrm{F}$ and assessing cell toxicity, cell proliferation, and osteogenic induction ability. The results showed a significant improvement in cell proliferation and enhancement of the firm attachment of the implant surface to the junction epithelium with a proper concentration of fluoride. Titanium alloy screws were functionalized with ultraviolet light and tested in animal experiments by Hirota et al. (100). The results showed that they could effectively increase the surface hydrophilicity, reduce pollution of carbonaceous matter, and form a positive conversion electrostatic charge, which played an important role in promoting the recruitment, adhesion, and proliferation of osteoblasts. Similar photo-functional surface treatments had also been reported to promote osteogenesis (101). However, the underlying mechanism is still unknown. Chika et al. (98) hypothesized that this phenomenon could be associated with a significant reduction in atomic carbon on titanium microfiber surfaces. Liu et al. (102) provided a partial insight into this; they found that with the accumulation of ultraviolet radiant energy, biological activities were increased step-by-step. The surface physicochemical changes induced by ultraviolet radiant energy alternated the functional site exposure of extracellular matrix proteins. They also demonstrated the synergistic effects of the FAKRhoA and ERK1/2 signaling pathways on mediating the URE-dependent cell behaviors.

Some physical treatments can also promote osteogenesis and inhibit bacterial growth. Magnetoelectric fields have been studied to promote bone tissue regeneration. Sahm et al. (103) reported that endogenous electric fields could alternate the direction of differentiation of stem cells. They used an alternating electric field stimulating human osteoblasts that were seeded on titanium electrodes and found that the expression of osteogenic-related proteins was significantly increased.

Moreover, the biological method was used to accelerate osteogenic procession. During the natural bone defect restoration process, stem cells (such as bone marrow mesenchymal stem cells) could differentiate into osteoblasts and osteoclasts, partly regulated by the exosome secretion of neighboring stem cells. Some researchers introduced early-staged osteogenic differentiation of stem cells into the titanium scaffold to demonstrate its osteogenic induction ability (104). Hong et al. (105) pretreated the surface of the 
titanium alloy with platelet-rich plasma, attached platelets to the surface of rough titanium alloy, and used the growth factor generated by the platelets to promote osteogenesis. The results reported by $\mathrm{Ma}$ et al. (106) may explain this phenomenon; they treated the $\mathrm{TiO}_{2}$ nanotubular surface with platelet-derived growth factor-BB and obtained similar osteogenic differentiation improvement results.

\section{Summary}

At present, titanium and its alloys are the most commonly used metals in bone tissue engineering. There has been considerable research on improving physical properties such as durability, corrosion resistance, elasticity, hardness, etc. These characteristics are the basis of its application. Considering the economic considerations, feasibility, and physical properties, it seems that the research has reached a bottleneck. Also, the biological activity of the titanium alloy still has significant room for improvement. Many studies have shown that there were still defects in the application of titanium and its alloys, and the mechanisms are still unknown.

At present, research into the improvement of the properties of titanium alloy bone tissue engineering has gradually transformed into research regarding the potential mechanisms. Although titanium is considered to have good bioactivity among metals, compared with other nonmetallic materials, such as biological ceramics and highmolecular polymers, it seems to exhibit no advantages. Of course, although organic materials have excellent biological activity, their physical properties still need to be improved. We cannot predict which kind of material will prevail in the future. It can be argued that improving the biological properties of metals is as important as improving the physical properties of organic materials. At the same time, the material-cell interactions on both sides are likely to be intricately connected aspects of the same mechanism.

In conclusion, although the research on improving the properties of titanium alloy bone tissue engineering may have entered a bottleneck stage, further research is still needed.

\section{Acknowledgments}

Funding: None.

\section{Footnote}

Reporting Checklist: The authors have completed the
Narrative Review reporting checklist. Available at https:// dx.doi.org/10.21037/atm-20-8175

Peer Review File: Available at https://dx.doi.org/10.21037/ atm-20-8175

Conflicts of Interest: All authors have completed the ICMJE uniform disclosure form (available at https://dx.doi. org/10.21037/atm-20-8175). The authors have no conflicts of interest to declare.

Ethical Statement: The authors are accountable for all aspects of the work in ensuring that questions related to the accuracy or integrity of any part of the work are appropriately investigated and resolved.

Open Access Statement: This is an Open Access article distributed in accordance with the Creative Commons Attribution-NonCommercial-NoDerivs 4.0 International License (CC BY-NC-ND 4.0), which permits the noncommercial replication and distribution of the article with the strict proviso that no changes or edits are made and the original work is properly cited (including links to both the formal publication through the relevant DOI and the license). See: https://creativecommons.org/licenses/by-nc-nd/4.0/.

\section{References}

1. Geetha M, Singh AK, Asokamani R, et al. Ti based biomaterials, the ultimate choice for orthopaedic implantsa review. Prog Mater Sci 2009;54:397-425.

2. Murr L, Esquivel E, Quinones S, et al. Microstructures and mechanical properties of electron beam-rapid manufactured Ti-6Al-4V biomedical prototypes compared to wrought Ti-6Al-4V. Mater Charact 2009;60:96-105.

3. Anselme K. Osteoblast adhesion on biomaterials. Biomaterials 2000;21:667-81.

4. Kunii T, Mori Y, Tanaka H, et al. Improved Osseointegration of a TiNbSn Alloy with a Low Young's Modulus Treated with Anodic Oxidation. Sci Rep 2019;9:13985.

5. Long $M$, Rack HJ. Titanium alloys in total joint replacement--a materials science perspective. Biomaterials 1998;19:1621-39.

6. Longhofer LK, Chong A, Strong NM, et al. Specific material effects of wear-particle-induced inflammation and osteolysis at the bone-implant interface: A rat model. J Orthop Translat 2017;8:5-11. 
7. Lian $\mathrm{F}$, Zhao $\mathrm{C}, \mathrm{Qu} \mathrm{J}$, et al. Icariin attenuates titanium particle-induced inhibition of osteogenic differentiation and matrix mineralization via miR-21-5p. Cell Biol Int 2018;42:931-9.

8. Bothe RT, Beaton LE, Davenport HA. Reaction of Bone to Multiple Metallic Implants. Surg Obstet Gynecol 1940;71:598-602.

9. Beder OE, Stevenson JK, Jones TW, et al. A further investigation of the surgical application of titanium metal in dogs. Surgery 1957;41:1012-5.

10. Brånemark PI, Breine U, Johansson B, et al. Regeneration of bone marrow. A clinical and experimental study following removal of bone marrow by curettage. Acta Anat (Basel) 1964;59:1-46.

11. Okazak Y, Nishimura E, Nakada H, et al. Surface analysis of Ti-15Zr-4Nb-4Ta alloy after implantation in rat tibia. Biomaterials 2001;22:599-607.

12. Yamaguchi S, Takadama H, Matsushita T, et al. Apatiteforming ability of Ti-15Zr-4Nb-4Ta alloy induced by calcium solution treatment. J Mater Sci Mater Med 2010;21:439-44.

13. Yamaguchi S, Takadama H, Matsushita T, et al. Preparation of bioactive Ti-15Zr-4Nb-4Ta alloy from $\mathrm{HCl}$ and heat treatments after an $\mathrm{NaOH}$ treatment. J Biomed Mater Res A 2011;97:135-44.

14. Okazaki Y, Nagata H. Comparisons of immersion and electrochemical properties of highly biocompatible Ti-15Zr-4Nb-4Ta alloy and other implantable metals for orthopedic implants. Sci Technol Adv Mater 2012;13:064216.

15. Blanquer A, Musilkova J, Barrios L, et al. Cytocompatibility assessment of Ti-Zr-Pd-Si-(Nb) alloys with low Young's modulus, increased hardness, and enhanced osteoblast differentiation for biomedical applications. J Biomed Mater Res B Appl Biomater 2018;106:834-42.

16. Liu $H$, Niinomi $M$, Nakai $M$, et al. $\beta$-Type titanium alloys for spinal fixation surgery with high Young's modulus variability and good mechanical properties. Acta Biomater 2015;24:361-9.

17. Kopova I, Stráský J, Harcuba P, et al. Newly developed Ti-Nb-Zr-Ta-Si-Fe biomedical beta titanium alloys with increased strength and enhanced biocompatibility. Mater Sci Eng C Mater Biol Appl 2016;60:230-8.

18. Huang R, Zhang L, Huang L, et al. Enhanced in-vitro osteoblastic functions on $\beta$-type titanium alloy using surface mechanical attrition treatment. Mater Sci Eng C Mater Biol Appl 2019;97:688-97.
19. Malinov S, Sha W, Voon CS, et al. In situ high temperature microscopy study of the surface oxidation and phase transformations in titanium alloys. J Microsc 2002;207:163-8.

20. Fu J, Liu X, Tan L, et al. Modulation of the mechanosensing of mesenchymal stem cells by laserinduced patterning for the acceleration of tissue reconstruction through the $\mathrm{Wnt} / \beta$-catenin signaling pathway activation. Acta Biomater 2020;101:152-67.

21. Tanase CE, Golozar M, Best SM, et al. Cell response to plasma electrolytic oxidation surface-modified low-modulus $\beta$-type titanium alloys. Colloids Surf B Biointerfaces 2019;176:176-84.

22. Xu X, Lu Y, Li S, et al. Copper-modified Ti6Al4V alloy fabricated by selective laser melting with pro-angiogenic and anti-inflammatory properties for potential guided bone regeneration applications. Mater Sci Eng C Mater Biol Appl 2018;90:198-210.

23. Liu X, Chen C, Zhang H, et al. Biocompatibility evaluation of antibacterial Ti-Ag alloys with nanotubular coatings. Int J Nanomedicine 2019;14:457-68.

24. Fernandez-Garcia E, Guillem-Marti J, GutierrezGonzalez CF, et al. Osteoblastic cell response to spark plasma-sintered zirconia/titanium cermets. J Biomater Appl 2015;29:813-23.

25. Kokubun $\mathrm{R}$, Wang $\mathrm{W}, \mathrm{Zhu} \mathrm{S}$, et al. In vivo evaluation of a Ti-based bulk metallic glass alloy bar. Biomed Mater Eng 2015;26:9-17.

26. Keller JC, Stanford CM, Wightman JP, et al. Characterizations of titanium implant surfaces. III. J Biomed Mater Res 1994;28:939-46.

27. Zheng G, Guan B, Hu P, et al. Topographical cues of direct metal laser sintering titanium surfaces facilitate osteogenic differentiation of bone marrow mesenchymal stem cells through epigenetic regulation. Cell Prolif 2018;51:e12460.

28. Zhu M, Ye H, Fang J, et al. Engineering High-Resolution Micropatterns Directly onto Titanium with Optimized Contact Guidance to Promote Osteogenic Differentiation and Bone Regeneration. ACS Appl Mater Interfaces 2019;11:43888-901.

29. Fujibayashi S, Neo M, Kim HM, et al. Osteoinduction of porous bioactive titanium metal. Biomaterials 2004;25:443-50.

30. Wang X, Schwartz Z, Gittens RA, et al. Role of integrin $\alpha 2 \beta 1$ in mediating osteoblastic differentiation on threedimensional titanium scaffolds with submicron-scale texture. J Biomed Mater Res A 2015;103:1907-18. 
31. Cheng A, Cohen DJ, Kahn A, et al. Laser Sintered Porous Ti-6Al-4V Implants Stimulate Vertical Bone Growth. Ann Biomed Eng 2017;45:2025-35.

32. García-Gareta E, Hua J, Orera A, et al. Biomimetic surface functionalization of clinically relevant metals used as orthopaedic and dental implants. Biomed Mater 2017;13:015008.

33. Ma L, Wang X, Zhao N, et al. Integrating 3D Printing and Biomimetic Mineralization for Personalized Enhanced Osteogenesis, Angiogenesis, and Osteointegration. ACS Appl Mater Interfaces 2018;10:42146-54.

34. Cheong VS, Fromme P, Coathup MJ, et al. Partial Bone Formation in Additive Manufactured Porous Implants Reduces Predicted Stress and Danger of Fatigue Failure. Ann Biomed Eng 2020;48:502-14.

35. Attar H, Löber L, Funk A, et al. Mechanical behavior of porous commercially pure $\mathrm{Ti}$ and $\mathrm{Ti}-\mathrm{TiB}$ composite materials manufactured by selective laser melting. Mater Sci Eng A 2015;625:350-6.

36. Wang H, Su K, Su L, et al. The effect of 3D-printed Ti6Al4V scaffolds with various macropore structures on osteointegration and osteogenesis: A biomechanical evaluation. J Mech Behav Biomed Mater 2018;88:488-96.

37. Hedayati R, Ahmadi SM, Lietaert K, et al. Isolated and modulated effects of topology and material type on the mechanical properties of additively manufactured porous biomaterials. J Mech Behav Biomed Mater 2018;79:254-63.

38. Zhou X, Zhang D, Wang M, et al. Three-Dimensional Printed Titanium Scaffolds Enhance Osteogenic Differentiation and New Bone Formation by Cultured Adipose Tissue-Derived Stem Cells Through the IGF1R/AKT/Mammalian Target of Rapamycin Complex 1 (mTORC1) Pathway. Med Sci Monit 2019;25:8043-54.

39. Nune KC, Kumar A, Murr LE, et al. Interplay between self-assembled structure of bone morphogenetic protein-2 (BMP-2) and osteoblast functions in three-dimensional titanium alloy scaffolds: Stimulation of osteogenic activity. J Biomed Mater Res A 2016;104:517-32.

40. Yang F, Chen C, Zhou Q, et al. Laser beam melting $3 \mathrm{D}$ printing of $\mathrm{Ti} 6 \mathrm{Al} 4 \mathrm{~V}$ based porous structured dental implants: fabrication, biocompatibility analysis and photoelastic study. Sci Rep 2017;7:45360.

41. Ran Q, Yang W, Hu Y, et al. Osteogenesis of 3D printed porous Ti6Al4V implants with different pore sizes. J Mech Behav Biomed Mater 2018;84:1-11.

42. Yu L, Wu Y, Liu J, et al. 3D Culture of Bone MarrowDerived Mesenchymal Stem Cells (BMSCs) Could
Improve Bone Regeneration in 3D-Printed Porous Ti6Al4V Scaffolds. Stem Cells Int 2018;2018:2074021.

43. Falkowska A, Seweryn A, Skrodzki M, et al. Strength Properties of a Porous Titanium Alloy Ti6Al4V with Diamond Structure Obtained by Laser Power Bed Fusion (LPBF). Materials (Basel) 2020. [Epub ahead of print]. doi:10.3390/ma13225138.

44. Kapat K, Srivas PK, Rameshbabu AP, et al. Influence of Porosity and Pore-Size Distribution in Ti6Al4 V Foam on Physicomechanical Properties, Osteogenesis, and Quantitative Validation of Bone Ingrowth by MicroComputed Tomography. ACS Appl Mater Interfaces 2017;9:39235-48.

45. Wang H, Zhao B, Liu C, et al. A Comparison of Biocompatibility of a Titanium Alloy Fabricated by Electron Beam Melting and Selective Laser Melting. PLoS One 2016;11:e0158513.

46. Qu YH, Li FL, Wen K, et al. Initial osteoblast functions on a type of near $\beta$-type titanium alloys surfaces modified by the double glow plasma nitriding technology. Zhonghua Kou Qiang Yi Xue Za Zhi 2017;52:132-6.

47. Shin YC, Pang KM, Han DW, et al. Enhanced osteogenic differentiation of human mesenchymal stem cells on $\mathrm{Ti}$ surfaces with electrochemical nanopattern formation. Mater Sci Eng C Mater Biol Appl 2019;99:1174-81.

48. Liu L, Zeng D, Chen Y, et al. Microarc oxidation surface of titanium implants promote osteogenic differentiation by activating ERK1/2-miR-1827-Osterix. In Vitro Cell Dev Biol Anim 2020;56:296-306.

49. Clainche TL, Linklater D, Wong S, et al. Mechano-Bactericidal Titanium Surfaces for Bone Tissue Engineering. ACS Appl Mater Interfaces 2020;12:48272-83.

50. Dumas V, Guignandon A, Vico L, et al. Femtosecond laser nano/micro patterning of titanium influences mesenchymal stem cell adhesion and commitment. Biomed Mater 2015;10:055002.

51. Gulati K, Prideaux M, Kogawa M, et al. Anodized 3D-printed titanium implants with dual micro- and nano-scale topography promote interaction with human osteoblasts and osteocyte-like cells. J Tissue Eng Regen Med 2017;11:3313-25.

52. Zhang J, Liu J, Wang C, et al. A comparative study of the osteogenic performance between the hierarchical micro/ submicro-textured 3D-printed Ti6Al4V surface and the SLA surface. Bioact Mater 2020;5:9-16.

53. Mróz W, Budner B, Syroka R, et al. In vivo implantation of porous titanium alloy implants coated with magnesium- 
doped octacalcium phosphate and hydroxyapatite thin films using pulsed laser depostion. J Biomed Mater Res B Appl Biomater 2015;103:151-8.

54. Jang YS, Moon SH, Nguyen TT, et al. In vivo bone regeneration by differently designed titanium membrane with or without surface treatment: a study in rat calvarial defects. J Tissue Eng 2019;10:2041731419831466.

55. Gnedenkov SV, Sinebryukhov SL, Puz AV, et al. In vivo study of osteogenerating properties of calcium-phosphate coating on titanium alloy Ti-6Al-4V. Biomed Mater Eng 2016;27:551-60.

56. Li K, Yan J, Wang C, et al. Graphene modified titanium alloy promote the adhesion, proliferation and osteogenic differentiation of bone marrow stromal cells. Biochem Biophys Res Commun 2017;489:187-92.

57. Chudinova EA, Surmeneva MA, Timin AS, et al. Adhesion, proliferation, and osteogenic differentiation of human mesenchymal stem cells on additively manufactured Ti6Al4V alloy scaffolds modified with calcium phosphate nanoparticles. Colloids Surf B Biointerfaces 2019;176:130-9.

58. Wang C, Hu H, Li Z, et al. Enhanced Osseointegration of Titanium Alloy Implants with Laser Microgrooved Surfaces and Graphene Oxide Coating. ACS Appl Mater Interfaces 2019;11:39470-83.

59. Li K, Xue Y, Zhou J, et al. Silanized NaCa2HSi3O9 nanorods with a reduced $\mathrm{pH}$ increase on $\mathrm{Ti}$ for improving osteogenesis and angiogenesis in vitro. J Mater Chem B 2020;8:691-702.

60. Li Q, Liang B, Wang F, et al. Delivery of Interleukin 4 from a Titanium Substrate Coated with Graphene Oxide for Enhanced Osseointegration by Regulating Macrophage Polarization. ACS Biomater Sci Eng 2020;6:5215-29.

61. Li Q, Wang Z. Involvement of FAK/P38 Signaling Pathways in Mediating the Enhanced Osteogenesis Induced by Nano-Graphene Oxide Modification on Titanium Implant Surface. Int J Nanomedicine 2020;15:4659-76.

62. Shi LY, Wang A, Zang FZ, et al. Tantalum-coated pedicle screws enhance implant integration. Colloids Surf B Biointerfaces 2017;160:22-32.

63. Wang F, Wang L, Feng Y, et al. Evaluation of an artificial vertebral body fabricated by a tantalum-coated porous titanium scaffold for lumbar vertebral defect repair in rabbits. Sci Rep 2018;8:8927.

64. Prinz C, Elhensheri M, Rychly J, et al. Antimicrobial and bone-forming activity of a copper coated implant in a rabbit model. J Biomater Appl 2017;32:139-49.
65. Yan Y, Wei Y, Yang R, et al. Enhanced osteogenic differentiation of bone mesenchymal stem cells on magnesium-incorporated titania nanotube arrays. Colloids Surf B Biointerfaces 2019;179:309-16.

66. Zhao S, Xu Y, Xu W, et al. Tremella-Like ZnO@Col-IDecorated Titanium Surfaces with Dual-Light-Defined Broad-Spectrum Antibacterial and Triple Osteogenic Properties. ACS Appl Mater Interfaces 2020;12:30044-51.

67. Cai X, Cai J, Ma K, et al. Fabrication and characterization of $\mathrm{Mg}$-doped chitosan-gelatin nanocompound coatings for titanium surface functionalization. J Biomater Sci Polym Ed 2016;27:954-71.

68. Saha S, Pramanik K, Biswas A, et al. Silk fibroin coated TiO2 nanotubes for improved osteogenic property of Ti6Al4V bone implants. Mater Sci Eng C Mater Biol Appl 2019;105:109982.

69. Haversath M, Hülsen T, Böge C, et al. Osteogenic differentiation and proliferation of bone marrowderived mesenchymal stromal cells on PDLLA + BMP2-coated titanium alloy surfaces. J Biomed Mater Res A 2016;104:145-54.

70. Labat B, Morin-Grognet S, Gaudière F, et al. Synergistic influence of topomimetic and chondroitin sulfate-based treatments on osteogenic potential of Ti-6Al-4V.J Biomed Mater Res A 2016;104:1988-2000.

71. Kim KJ, Itoh T, Kotake S, et al. Effects of recombinant human bone morphogenetic protein-2 on human bone marrow cells cultured with various biomaterials. J Biomed Mater Res 1997;35:279-85.

72. Lv J, Xiu P, Tan J, et al. Enhanced angiogenesis and osteogenesis in critical bone defects by the controlled release of BMP-2 and VEGF: implantation of electron beam melting-fabricated porous Ti6Al4V scaffolds incorporating growth factor-doped fibrin glue. Biomed Mater 2015;10:035013.

73. Huang L, Luo Z, Hu Y, et al. Enhancement of local bone remodeling in osteoporotic rabbits by biomimic multilayered structures on Ti6Al4V implants. J Biomed Mater Res A 2016;104:1437-51.

74. Teng FY, Tai IC, Ho ML, et al. Controlled release of BMP-2 from titanium with electrodeposition modification enhancing critical size bone formation. Mater Sci Eng C Mater Biol Appl 2019;105:109879.

75. Guillot R, Pignot-Paintrand I, Lavaud J, et al. Assessment of a polyelectrolyte multilayer film coating loaded with BMP-2 on titanium and PEEK implants in the rabbit femoral condyle. Acta Biomater 2016;36:310-22.

76. Göttlicher M, Rohnke M, Moryson Y, et al. 
Functionalization of Ti-40Nb implant material with strontium by reactive sputtering. Biomater Res 2017;21:18.

77. Huang D, Ma K, Cai X, et al. Evaluation of antibacterial, angiogenic, and osteogenic activities of green synthesized gap-bridging copper-doped nanocomposite coatings. Int J Nanomedicine 2017;12:7483-500.

78. Ankha MDVEA, Silva AM, Prado RFD, et al. Effect of DLC Films with and without Silver Nanoparticles Deposited On Titanium Alloy. Braz Dent J 2019;30:607-16.

79. Wang X, Yan L, Ye T, et al. Osteogenic and antiseptic nanocoating by in situ chitosan regulated electrochemical deposition for promoting osseointegration. Mater Sci Eng C Mater Biol Appl 2019;102:415-26.

80. van Hengel IAJ, Putra NE, Tierolf MWAM, et al. Biofunctionalization of selective laser melted porous titanium using silver and zinc nanoparticles to prevent infections by antibiotic-resistant bacteria. Acta Biomater 2020;107:325-37.

81. Liu D, He C, Liu Z, et al. Gentamicin coating of nanotubular anodized titanium implant reduces implantrelated osteomyelitis and enhances bone biocompatibility in rabbits. Int J Nanomedicine 2017;12:5461-71.

82. Liu P, Hao Y, Zhao Y, et al. Surface modification of titanium substrates for enhanced osteogenetic and antibacterial properties. Colloids Surf B Biointerfaces 2017;160:110-6.

83. Pura JA, Bobyn JD, Tanzer M, et al. Implant-delivered Alendronate Causes a Dose-dependent Response on Net Bone Formation Around Porous Titanium Implants in Canines. Clin Orthop Relat Res 2016;474:1224-33.

84. Rojo L, Gharibi B, McLister R, et al. Self-assembled monolayers of alendronate on Ti6Al4V alloy surfaces enhance osteogenesis in mesenchymal stem cells. Sci Rep 2016;6:30548.

85. Xiu P, Jia Z, Lv J, et al. Tailored Surface Treatment of 3D Printed Porous Ti6Al4V by Microarc Oxidation for Enhanced Osseointegration via Optimized Bone InGrowth Patterns and Interlocked Bone/Implant Interface. ACS Appl Mater Interfaces 2016;8:17964-75.

86. Chen M, Huang L, Shen X, et al. Construction of multilayered molecular reservoirs on a titanium alloy implant for combinational drug delivery to promote osseointegration in osteoporotic conditions. Acta Biomater 2020;105:304-18.

87. Zhai M, Zhu Y, Yang M, et al. Human Mesenchymal Stem Cell Derived Exosomes Enhance Cell-Free Bone Regeneration by Altering Their miRNAs Profiles. Adv Sci (Weinh) 2020;7:2001334.
88. Lan Y, Jin Q, Xie H, et al. Exosomes Enhance Adhesion and Osteogenic Differentiation of Initial Bone Marrow Stem Cells on Titanium Surfaces. Front Cell Dev Biol 2020;8:583234.

89. Li Y, Yang W, Li X, et al. Improving osteointegration and osteogenesis of three-dimensional porous Ti6Al4V scaffolds by polydopamine-assisted biomimetic hydroxyapatite coating. ACS Appl Mater Interfaces 2015;7:5715-24.

90. Zhang Y, Wang F, Huang Q, et al. Layer-by-layer immobilizing of polydopamine-assisted $\varepsilon$-polylysine and gum Arabic on titanium: Tailoring of antibacterial and osteogenic properties. Mater Sci Eng C Mater Biol Appl 2020;110:110690.

91. Meng Y, Li X, Li Z, et al. Surface Functionalization of Titanium Alloy with miR-29b Nanocapsules To Enhance Bone Regeneration. ACS Appl Mater Interfaces 2016;8:5783-93.

92. Atluri K, Lee J, Seabold D, et al. Gene-Activated Titanium Surfaces Promote In Vitro Osteogenesis. Int J Oral Maxillofac Implants 2017;32:e83-e96.

93. Liu H, Li W, Liu C, et al. Incorporating simvastatin/ poloxamer 407 hydrogel into 3D-printed porous Ti6Al4V scaffolds for the promotion of angiogenesis, osseointegration and bone ingrowth. Biofabrication 2016;8:045012.

94. Man Z, Sha D, Sun S, et al. In Vitro Bioactivity Study of RGD-Coated Titanium Alloy Prothesis for Revision Total Hip Arthroplasty. Biomed Res Int 2016;2016:8627978.

95. Hanawa T, Ukai H, Murakami K. X-ray photoelectron spectroscopy of calcium-ion-implanted titanium. J Electron Spectros Relat Phenomena 1993;63:347-54.

96. Su Y, Komasa S, Li P, et al. Synergistic effect of nanotopography and bioactive ions on peri-implant bone response. Int J Nanomedicine 2017;12:925-34.

97. Cazzola M, Ferraris S, Boschetto F, et al. Green Tea Polyphenols Coupled with a Bioactive Titanium Alloy Surface: In Vitro Characterization of Osteoinductive Behavior through a KUSA A1 Cell Study. Int J Mol Sci 2018;19:2255.

98. Iwasaki C, Hirota M, Tanaka M, et al. Tuning of Titanium Microfiber Scaffold with UV-Photofunctionalization for Enhanced Osteoblast Affinity and Function. Int J Mol Sci 2020;21:738.

99. Pham MH, Haugen HJ, Rinna A, et al. Hydrofluoric acid treatment of titanium surfaces enhances the proliferation of human gingival fibroblasts. J Tissue Eng 2019;10:2041731419828950. 
100. Hirota M, Tanaka M, Ishijima M, et al. Effect of Photofunctionalization on Ti6Al4V Screw Stability Placed in Segmental Bone Defects in Rat Femurs. J Oral Maxillofac Surg 2016;74:861.e1-16.

101. Tabuchi M, Ikeda T, Hirota M, et al. Effect of UV Photofunctionalization on Biologic and Anchoring Capability of Orthodontic Miniscrews. Int J Oral Maxillofac Implants 2015;30:868-79.

102.Liu C, Sun M, Wang Y, et al. Ultraviolet Radiant EnergyDependent Functionalization Regulates Cellular Behavior on Titanium Dioxide Nanodots. ACS Appl Mater Interfaces 2020;12:31793-803.

103.Sahm F, Ziebart J, Jonitz-Heincke A, et al. Alternating Electric Fields Modify the Function of Human Osteoblasts Growing on and in the Surroundings of Titanium Electrodes. Int J Mol Sci 2020. [Epub ahead of print]. doi:10.3390/ijms21186944.

Cite this article as: Zuo W, Yu L, Lin J, Yang Y, Fei Q. Properties improvement of titanium alloys scaffolds in bone tissue engineering: a literature review. Ann Transl Med 2021;9(15):1259. doi: 10.21037/atm-20-8175
104. García-Gareta E, Hua J, Blunn GW, et al. Osseointegration of acellular and cellularized osteoconductive scaffolds: is tissue engineering using mesenchymal stem cells necessary for implant fixation? J Biomed Mater Res A 2015;103:1067-76.

105.Hong SH, Nam J, Kim HJ, et al. Platelet-Rich Plasma Pretreatment on Grit-Blasted Titanium Alloy for Enhanced Osteogenic Differentiation of Human Adipose-Derived Stem Cells. Clin Orthop Surg 2019;11:361-8.

106.Ma Q, Jiang N, Liang S, et al. Functionalization of a clustered $\mathrm{TiO} 2$ nanotubular surface with platelet derived growth factor-BB covalent modification enhances osteogenic differentiation of bone marrow mesenchymal stem cells. Biomaterials 2020;230:119650.

(English Language Editors: A. Kassem and J. Chapnick) 